\title{
Morphology Optimization Method for Machined Surface of Hardened Steel
}

\author{
SongtaoWang ${ }^{1}$, Yongda Yan $^{2}$, Bin Jiang ${ }^{3}$, MinliZheng ${ }^{4}$ and Yihang Fan ${ }^{5}$ \\ ${ }^{1,2}$ School of Mechanical and Electrical Engineering, Harbin Institute of \\ Technology, Harbin51\#, 150066, China, \\ ${ }^{3,4,5}$ School of Mechanical and Power Engineering, Harbin University of science \\ and technology, Harbin, 150080, China \\ hlgwst@sina.com
}

\begin{abstract}
The work analyzed the elements of high-speed cutting on hardened steel with hierarchy analysis method, studying the relational structure characteristics of control variables and performance indicators in high-speed cutting. And the hierarchy of evaluation indicator in high-speed cutting of hardened steel was proposed. Besides, the morphology, controlling flow of machined surface, was studied in high-speed cutting of hardened steel, proposing the criterion for surface roughness and surface texture and developing the methods of morphology prediction and control on machined surface. Then, validation experiments were conducted on hardened-steel dies of typical automotive panel to evaluate the accuracy and applicability of morphology forecasting and control methods.
\end{abstract}

Keywords: hardened steel, morphology, optimization

\section{Introduction}

There are many factors on the morphology of machined free-form surface in high-speed cutting of hardened steel, and they have different degrees of effects on the morphology. There are complex interacting laws between these various factors. The optimization order and optimization methods of different parts, based on their technological characteristics and requirements, need to be determined to improve the surface quality of parts. Currently, many scholars have conducted intensive researches on the quality optimization for the machined surface of work-pieces. Yan established the surface generation modeland morphology simulation algorithm ofball-end cutting[1]. Chen analyzed and simplified the geometrical and physical factors, establishing a new mathematical model of surface roughness and contour in high-speed cutting [2]. Feng established computer simulation system for the morphology of axial cutting [3]. Gaostudied the cutting surface morphology and end-cutting deformation by simulation [4]. Chenproposed the simulation system model of surface roughness [5]. Yu studied several $3 \mathrm{D}$-assessment parameters of surface micro-contour, proposing the corresponding mathematical model [6]. Li presented an on line-identifying model for fuzzy neural network of surface roughness [7]. Ge calculated its fractal dimension through conventional contour graph and appropriate calculation method [8]. Li utilized the concepts of contour spectral moment and surface spectral moment for fractal characterization of roughness assessment parameters, putting forward new 3D assessment parameters on this basis [9]. He studied the effect of the matching degree between surface roughness and hardness on contact fatigue properties [10]. Zhu studied the texture characteristics and friction characteristics of work-pieces surface in cutting process, concluding the relationship between surface morphology and surface properties [11].

Interpretative structural modeling was applied to solving the hierarchy between all the elements of high-speed cutting on free-form surface of hardened steel, establishing a 
hierarchical model of these processing elements. Prediction and control methods of surface morphology were proposed, and validation experiments were carried out on hardened steel dies of typical automotive panel.

\section{Element Set and Relationship Set in High-speed Cutting of Hardened Steel}

Interpretative structural modeling can reveal the internal structure of system to analyze the relational structurebetween complex system elements based on the disordered relationship and system elements [12]. The goals of parameter optimization are not clear due to the interaction between various elements.Interpretative structural modeling can solve the hierarchical relationships between various cutting elements, optimizing the sequence of parameter optimization.

The elements that set in high-speed cutting of hardened steel are that spindle speed is $n$, feed per tooth is $f z$,row spacing is ae, processing inclination angle is $\lambda$, cutting temperature is $t$, cutting force is $F$, vibration amplitude and frequency of cutting is that $A$ adds $f$, the life of tools is $T$, surface contour tolerance is $T m$, surface size tolerance is $T c$, the surface roughness in row direction is $R_{a}^{a e}$, the surface roughness in feeding direction is $R_{a}^{f}$, the uniformity of surface roughness is $\Delta R_{a}$, the uniformity of surface texture is $\Delta l$, the texture direction of the surface is $\phi_{w}$, and the processing efficiency is $V$, and all the elements are indicated with $\operatorname{Si}(i=1,2, \ldots, 16)$.

The establishment of relationship set in high-speed cutting of hardened steel. The binary correlation chart of cutting elements was established, where 1 means row element has a direct restriction with the rank element and Omeans no direct relationship between them. 
Table 1. Binary Correlation Chartof the Elements in High-speed Cutting of Hardened Steel

\begin{tabular}{c|c|c|c|c|c|c|c|c|c|c|c|c|c|c|c|c}
\hline & $S_{1}$ & $S_{2}$ & $S_{3}$ & $S_{4}$ & $S_{5}$ & $S_{6}$ & $S_{7}$ & $S_{8}$ & $S_{9}$ & $S_{10}$ & $S_{11}$ & $S_{12}$ & $S_{13}$ & $S_{14}$ & $S_{15}$ & $S_{16}$ \\
\hline$S_{1}$ & 1 & 0 & 0 & 0 & 1 & 1 & 1 & 0 & 0 & 0 & 0 & 0 & 0 & 0 & 0 & 1 \\
\hline$S_{2}$ & 0 & 1 & 0 & 0 & 1 & 1 & 0 & 0 & 0 & 0 & 0 & 1 & 1 & 0 & 1 & 1 \\
\hline$S_{3}$ & 0 & 0 & 1 & 0 & 1 & 1 & 0 & 0 & 0 & 0 & 1 & 0 & 1 & 0 & 1 & 1 \\
\hline$S_{4}$ & 0 & 0 & 0 & 1 & 1 & 1 & 0 & 0 & 0 & 0 & 0 & 0 & 0 & 0 & 0 & 0 \\
\hline$S_{5}$ & 0 & 0 & 0 & 0 & 1 & 1 & 0 & 1 & 0 & 0 & 0 & 0 & 0 & 0 & 0 & 0 \\
\hline$S_{6}$ & 0 & 0 & 0 & 0 & 1 & 1 & 1 & 1 & 0 & 0 & 0 & 0 & 0 & 0 & 0 & 0 \\
\hline$S_{7}$ & 0 & 0 & 0 & 0 & 0 & 0 & 1 & 1 & 1 & 1 & 0 & 1 & 1 & 1 & 1 & 0 \\
\hline$S_{8}$ & 0 & 0 & 0 & 0 & 0 & 0 & 0 & 1 & 1 & 1 & 0 & 0 & 0 & 0 & 0 & 1 \\
\hline$S_{9}$ & 0 & 0 & 0 & 0 & 0 & 0 & 0 & 0 & 1 & 0 & 0 & 0 & 0 & 0 & 0 & 1 \\
\hline$S_{10}$ & 0 & 0 & 0 & 0 & 0 & 0 & 0 & 0 & 0 & 1 & 0 & 0 & 0 & 0 & 0 & 1 \\
\hline$S_{11}$ & 0 & 0 & 0 & 0 & 0 & 0 & 0 & 0 & 0 & 0 & 1 & 0 & 1 & 0 & 0 & 1 \\
\hline$S_{12}$ & 0 & 0 & 0 & 0 & 0 & 0 & 0 & 0 & 0 & 0 & 0 & 1 & 1 & 0 & 0 & 1 \\
\hline$S_{13}$ & 0 & 0 & 0 & 0 & 0 & 0 & 0 & 0 & 0 & 0 & 0 & 0 & 1 & 0 & 0 & 1 \\
\hline$S_{14}$ & 0 & 0 & 0 & 0 & 0 & 0 & 0 & 0 & 0 & 0 & 0 & 0 & 0 & 1 & 0 & 1 \\
\hline$S_{15}$ & 0 & 0 & 0 & 0 & 0 & 0 & 0 & 0 & 0 & 0 & 0 & 0 & 0 & 0 & 1 & 1 \\
\hline$S_{16}$ & 0 & 0 & 0 & 0 & 0 & 0 & 0 & 0 & 0 & 0 & 0 & 0 & 0 & 0 & 0 & 1 \\
\hline
\end{tabular}

Adjacent matrix $A$, according to binary correlation chart, can be built as follows.

$$
A=\left|\begin{array}{llllllllllllllll}
1 & 0 & 0 & 0 & 1 & 1 & 1 & 0 & 0 & 0 & 0 & 0 & 0 & 0 & 0 & 1 \\
0 & 1 & 0 & 0 & 1 & 1 & 0 & 0 & 0 & 0 & 0 & 1 & 1 & 0 & 1 & 1 \\
0 & 0 & 1 & 0 & 1 & 1 & 0 & 0 & 0 & 0 & 1 & 0 & 1 & 0 & 1 & 1 \\
0 & 0 & 0 & 1 & 1 & 1 & 0 & 0 & 0 & 0 & 0 & 0 & 0 & 0 & 0 & 0 \\
0 & 0 & 0 & 0 & 1 & 1 & 0 & 1 & 0 & 0 & 0 & 0 & 0 & 0 & 0 & 0 \\
0 & 0 & 0 & 0 & 1 & 1 & 1 & 1 & 0 & 0 & 0 & 0 & 0 & 0 & 0 & 0 \\
0 & 0 & 0 & 0 & 0 & 0 & 1 & 1 & 1 & 1 & 0 & 1 & 1 & 1 & 1 & 0 \\
0 & 0 & 0 & 0 & 0 & 0 & 0 & 1 & 1 & 1 & 0 & 0 & 0 & 0 & 0 & 1 \\
0 & 0 & 0 & 0 & 0 & 0 & 0 & 0 & 1 & 0 & 0 & 0 & 0 & 0 & 0 & 1 \\
0 & 0 & 0 & 0 & 0 & 0 & 0 & 0 & 0 & 1 & 0 & 0 & 0 & 0 & 0 & 1 \\
0 & 0 & 0 & 0 & 0 & 0 & 0 & 0 & 0 & 0 & 1 & 0 & 1 & 0 & 0 & 1 \\
0 & 0 & 0 & 0 & 0 & 0 & 0 & 0 & 0 & 0 & 0 & 1 & 1 & 0 & 0 & 1 \\
0 & 0 & 0 & 0 & 0 & 0 & 0 & 0 & 0 & 0 & 0 & 0 & 1 & 0 & 0 & 1 \\
0 & 0 & 0 & 0 & 0 & 0 & 0 & 0 & 0 & 0 & 0 & 0 & 0 & 1 & 0 & 1 \\
0 & 0 & 0 & 0 & 0 & 0 & 0 & 0 & 0 & 0 & 0 & 0 & 0 & 0 & 1 & 1 \\
0 & 0 & 0 & 0 & 0 & 0 & 0 & 0 & 0 & 0 & 0 & 0 & 0 & 0 & 0 & 1
\end{array}\right|
$$

Thesolvingsteps for the reach abilitymatrixofmatrixfellows.

$$
R^{(n)}=A \circ A \circ \cdots \circ A=A^{(n-1)} \circ A(1)
$$

$R^{(n)}$ is called the reachability matrix of system factors, if $R^{(n-1)} \neq R^{(n)}$ and $R^{(n)}=R^{(n+1)}$, denoted by $\mathrm{R}$.

$$
R=\left(r_{i j}\right)=I \circ A \circ A \circ \cdots \circ A=(I \circ A)^{n}
$$

The relationship set of hardened-steel cutting system can be obtained through solving the reach abilitymatrix with formula2. The reachability matrix $R$ is fellow. 


\section{Hierarchy of Processing Elements in High-speed Cutting of Hardened Steel}

The hierarchy ofhardened-steel machining systemwas divided through the follow steps.

(1) Throughthe division for the hierarchy of system elements, we solve the reachability set $R\left(S_{i}\right)$ and antecedent set $A\left(S_{j}\right)$ of element $S_{i}$. The meanings of $R\left(S_{i}\right)$ and $A\left(S_{j}\right)$ are fellows.
$A\left(S_{i}\right)=\left\{\right.$ all the elements that reach to element $\left.S_{j}\right\}$
$=\left\{\right.$ the elements that have strong correlation with $S_{i}$ in the next hierarchy $\}$
$=\{$ the corresponding row elements of all the element 1 at $j$ rank in reachability matrix $\}$ $R\left(S_{i}\right)=\left\{\right.$ all the elements that are in the same hierarchy with $S_{i}$ and also have strong correlation with it $\}$ $=\{$ the corresponding rank elements of all the element 1 at $i$ row in reachability matrix $\mathrm{R}\}$

(2) The method of hierarchical division is that $L_{0}$ equals $\varphi$ (null set), the set $L_{1}, L_{2} \ldots, L_{k}$ indicates a hierarchy from the top down, then:

$$
\pi_{L}(S)=\left[L_{1}, L_{2}, \cdots, L_{k}\right]
$$

Thealgorithm of $L_{k}$ isfellow.

$$
L_{k}=\left\{S_{i} \in S-L_{0}-\cdots-L_{k-1}\left(S_{i}\right) / R_{k-1}\left(S_{i}\right) \text { I } A_{k-1}\left(S_{i}\right)=R_{k-1}\left(S_{i}\right)\right\}(4)
$$

The dividing steps on the elements in high-speed cutting of hardened steel are shown in table 2. The elements $S_{i}(i=1,2, \cdots 15)$ are indicated with numbers $i(i=1,2, \cdots 15)$ for convenience in the Table2.

Table 2. Division Calculation Table for High-speed Cutting Elements of Hardened Steel

\begin{tabular}{c|c|c|c}
\hline$S i$ & $R(S i)$ & $A(S i)$ & $R(S i) \cap A(\mathrm{Si})$ \\
\hline $\mathrm{S}_{1}$ & $\{1,5,6,7,8,9,10,12,13,14,15,16\}$ & $\{1\}$ & $\{1\}$ \\
\hline $\mathrm{S}_{2}$ & $\{2,5,6,7,8,9,10,12,13,14,15,16\}$ & $\{2\}$ & $\{2\}$ \\
\hline $\mathrm{S}_{3}$ & $\{3,5,6,7,8,9,10,11,12,13,14,15,16\}$ & $\{3\}$ & $\{3\}$ \\
\hline $\mathrm{S}_{4}$ & $\{4,5,6,7,8,9,10,12,13,14,15,16\}$ & $\{4\}$ & $\{4\}$ \\
\hline $\mathrm{S}_{5}$ & $\{5,6,7,8,9,10,12,13,14,15,16\}$ & $\{1,2,3,4,5,6\}$ & $\{5,6\}$ \\
\hline $\mathrm{S}_{6}$ & $\{5,6,7,8,9,10,12,13,14,15,16\}$ & $\{1,2,3,4,5,6\}$ & $\{5,6\}$ \\
\hline $\mathrm{S}_{7}$ & $\{7,8,9,10,12,13,14,15,16\}$ & $\{1,2,3,4,5,6,7\}$ & $\{7\}$ \\
\hline $\mathrm{S}_{8}$ & $\{8,9,10,16\}$ & $\{1,2,3,4,5,6,7,8\}$ & $\{8\}$ \\
\hline
\end{tabular}




\begin{tabular}{c|c|c|c}
\hline $\mathrm{S}_{9}$ & $\{9,16\}$ & $\{1,2,3,4,5,6,7,8,9\}$ & $\{9\}$ \\
\hline $\mathrm{S}_{10}$ & $\{10,16\}$ & $\{1,2,3,4,5,6,7,8,10\}$ & $\{10\}$ \\
\hline $\mathrm{S}_{11}$ & $\{11,13,16\}$ & $\{3,11\}$ & $\{11\}$ \\
\hline $\mathrm{S}_{12}$ & $\{12,13,16\}$ & $\{1,2,3,4,5,6,7,12\}$ & $\{12\}$ \\
\hline $\mathrm{S}_{13}$ & $\{13,16\}$ & $\{1,2,3,4,5,6,7,11,12,13\}$ & $\{13\}$ \\
\hline $\mathrm{S}_{14}$ & $\{14,16\}$ & $\{1,2,3,4,5,6,7,14\}$ & $\{14\}$ \\
\hline $\mathrm{S}_{15}$ & $\{15,16\}$ & $\{1,2,3,4,5,6,7,15\}$ & $\{15\}$ \\
\hline $\mathrm{S}_{16}$ & $\{16\}$ & $\{1,2,3,4,5,6,7,8,9,10,11,1$ & $\{16\}$ \\
\hline
\end{tabular}

Thus the first hierarchy of element set is that $L_{1}$ equals $\{16\}$, and the comprehensive division of hierarchy can be obtained by repeating this method.

$\pi_{L}(S)=\left[L_{1}, L_{2}, \cdots, L_{7}\right]=\left[\left\{S_{16}\right\},\left\{S_{9}, S_{10}, S_{13}, S_{14}, S_{15}\right\},\left\{S_{8}, S_{11}, S_{12}\right\},\left\{S_{7}\right\},\left\{S_{5}, S_{6}\right\},\left\{S_{1}, S_{2}, S_{3}, S_{4}\right\}\right]$

The strong-connection table of cutting element, after the division, is shown in Table 3.

Table 3. Strong-connection Table for High-speed Cutting Elements of Hardened Steel

\begin{tabular}{ccccccccccccccccc}
\hline & $S_{16}$ & $S_{9}$ & $S_{10}$ & $S_{13}$ & $S_{14}$ & $S_{15}$ & $S_{8}$ & $S_{11}$ & $S_{12}$ & $S_{7}$ & $S_{5}$ & $S_{6}$ & $S_{1}$ & $S_{2}$ & $S_{3}$ & $S_{4}$ \\
$S_{16}$ & 1 & 0 & 0 & 0 & 0 & 0 & 0 & 0 & 0 & 0 & 0 & 0 & 0 & 0 & 0 & 0 \\
$S_{9}$ & 1 & 1 & 0 & 0 & 0 & 0 & 0 & 0 & 0 & 0 & 0 & 0 & 0 & 0 & 0 & 0 \\
$S_{10}$ & 1 & 0 & 1 & 0 & 0 & 0 & 0 & 0 & 0 & 0 & 0 & 0 & 0 & 0 & 0 & 0 \\
$S_{13}$ & 1 & 0 & 0 & 1 & 0 & 0 & 0 & 0 & 0 & 0 & 0 & 0 & 0 & 0 & 0 & 0 \\
$S_{14}$ & 1 & 0 & 0 & 0 & 1 & 0 & 0 & 0 & 0 & 0 & 0 & 0 & 0 & 0 & 0 & 0 \\
$S_{15}$ & 1 & 0 & 0 & 0 & 0 & 1 & 0 & 0 & 0 & 0 & 0 & 0 & 0 & 0 & 0 & 0 \\
$S_{8}$ & 1 & 1 & 1 & 0 & 0 & 0 & 1 & 0 & 0 & 0 & 0 & 0 & 0 & 0 & 0 & 0 \\
$S_{11}$ & 1 & 0 & 0 & 1 & 0 & 0 & 0 & 1 & 0 & 0 & 0 & 0 & 0 & 0 & 0 & 0 \\
$S_{12}$ & 1 & 0 & 0 & 1 & 0 & 0 & 0 & 0 & 1 & 0 & 0 & 0 & 0 & 0 & 0 & 0 \\
$S_{7}$ & 1 & 1 & 1 & 1 & 1 & 1 & 1 & 0 & 1 & 1 & 0 & 0 & 0 & 0 & 0 & 0 \\
$S_{5}$ & 1 & 1 & 1 & 1 & 1 & 1 & 1 & 0 & 1 & 1 & 1 & 1 & 0 & 0 & 0 & 0 \\
$S_{6}$ & 1 & 1 & 1 & 1 & 1 & 1 & 1 & 0 & 1 & 1 & 1 & 1 & 0 & 0 & 0 & 0 \\
$S_{1}$ & 1 & 1 & 1 & 1 & 1 & 1 & 1 & 0 & 1 & 1 & 1 & 1 & 1 & 0 & 0 & 0 \\
$S_{2}$ & 1 & 1 & 1 & 1 & 1 & 1 & 1 & 0 & 1 & 1 & 1 & 1 & 0 & 1 & 0 & 0 \\
$S_{3}$ & 1 & 1 & 1 & 1 & 1 & 1 & 1 & 1 & 1 & 1 & 1 & 1 & 0 & 0 & 1 & 0 \\
$S_{4}$ & 1 & 1 & 1 & 1 & 1 & 1 & 1 & 0 & 1 & 1 & 1 & 1 & 0 & 0 & 0 & 1 \\
\hline
\end{tabular}

Thus, the hierarchy of basic variables in high-speed cutting of hardened steel has been divided. However, initial cutting conditions also need to be determined. The vibration parameters, in cutting process, have direct influence on the vibration performance of the cutting tool. Die parameters (such as hardness and surface structure type of the work-pieces) and the structure of the cutting tool determine the cutting performance by changing the cutting temperature and force. Initial cutting conditions are hardness of the work-pieces, curvature radius of the work-pieces in spacing direction, curvature radius of the work-pieces in feeding direction, tool diameter, the teeth number of the tool, theintertooth space, the cutting edge form, the extended length, the vibration performance and the tool path. The hierarchy of variable elements in high-speed cutting of hardened steel can be drawn according to the hierarchy division and initial conditions, were shown in Figure 1. 


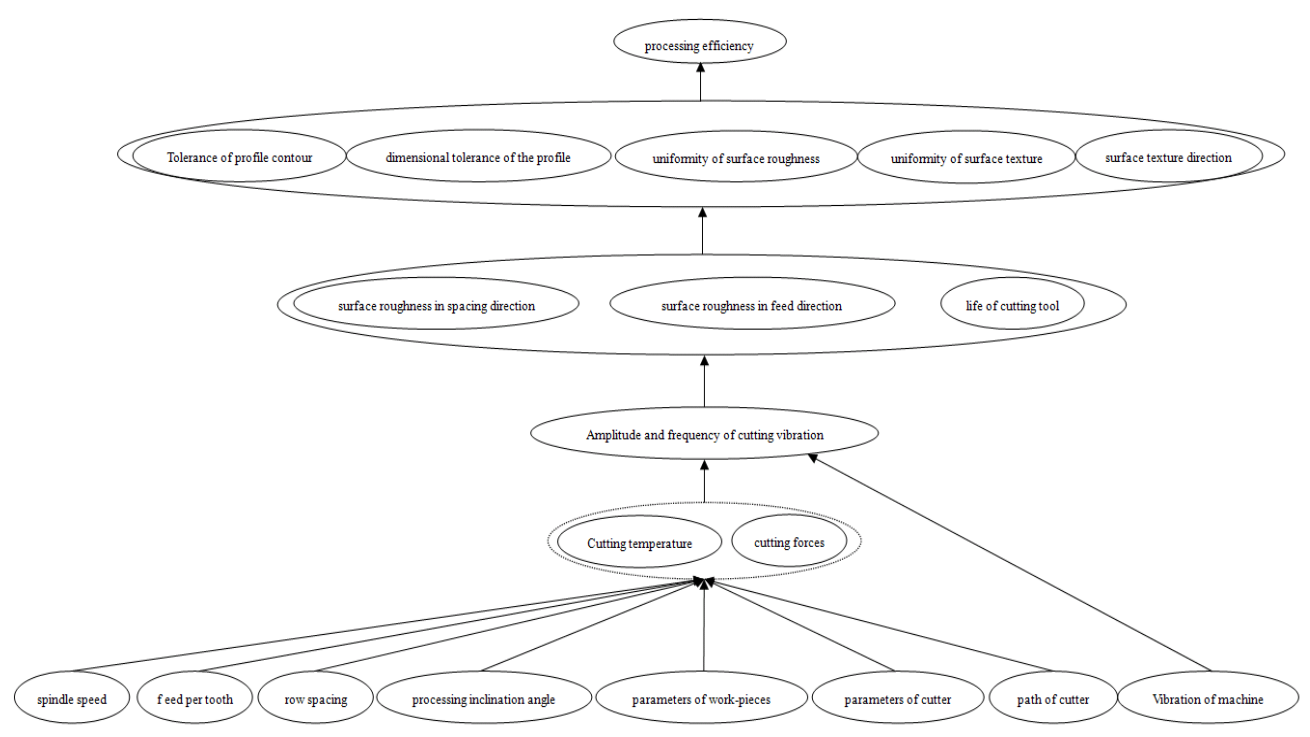

Figure 1. Hierarchy Chart of Variable Elements in High-speed Cutting of Hardened Steel

The sequences of element optimization arefellows.

(1)Processing efficiency;

(2)The tolerance of profile contour, dimensional tolerance of the profile, uniformity of surface roughness, uniformity of surface texture, surface texture direction;

(3)The surface roughness in spacing direction, the surface roughness in feed direction, the life of cutting tool;

(4) Amplitude and frequency of cutting vibration;

(5) Cutting temperature, cutting forces.

\section{Control Methods for Machined Surface Morphology of Hardened Steel}

\subsection{Criterion of Machined SurfaceMorphology}

Surface morphology includes surface roughness and the surfacetexture of work-pieces in high-speed cutting of hardened steel. These elements determine the surface quality and affect the service life of work-pieces.

4.1.1 Surface Roughness Model of High Speed Cutting:Surface roughness is divided into two parts, $R_{a}^{\prime}$ is surface roughness infeed direction and $R_{a}^{a}$ is surface roughness in row spacing direction, which can be obtained by simulation or experiment. The uniformity of surface roughness can describe the surface roughness distribution in two directions and maintain a uniform stress distribution on work-pieces surface under working conditions, extending for its service life. The uniformity degree of surface roughness is equal to the maximum difference between $R_{a}^{\prime}$ and $R_{a}^{a c}$ (refer to Formula 5).

$$
\Delta R_{a}=\max \left(\left|R_{a}^{f}-R_{a}^{a c}\right|\right)(5)
$$

Constraint conditions are fellows. 
$\left\{\begin{aligned} R_{a}^{f} & \leq R_{a \max } \\ R_{a}^{a e} & \leq R_{a \max } \\ \Delta R_{a} & \leq \Delta R_{a \max }\end{aligned}(6)\right.$

Where $R_{\text {amax }}$ is the maximum surface roughness in practical production; $\Delta R_{\text {amax }}$ is the maximum uniformity of surface roughness in practical production.

4.1.2. Surface Texture Model of High Speed Cutting:Surface texture determines the time of polishing operation. Meanwhile, the direction and uniformity of surface texture determine the stress distribution on each part of work-pieces surface and affect its service life.

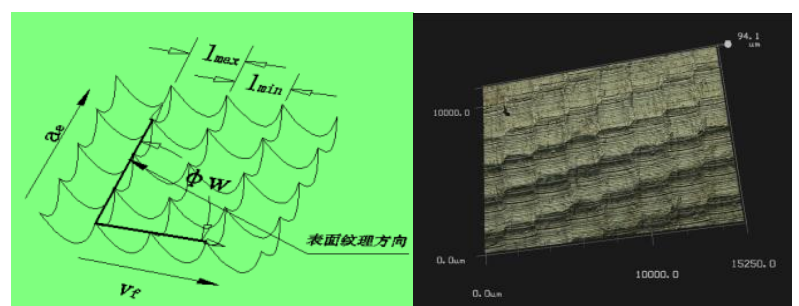

\section{Figure 2. Simulation and Test Results of Surface Texture}

The arrangement of surface micro-unit, was shown in figure2, forms the micro-morphology and surface texture. And the direction with the most obvious texture of the direction with the deepest groove is the surface texturedirection. Because, in texture direction, the vertical distance between the lowest and highest points of the groove is larger than that in other directions. That is the follow figure.

$$
h_{w}>h_{r}
$$

Where $h_{w}$ is the vertical distance between the lowest and highest point of the groove on the work-pieces surface in texture direction; $h_{r}$ is the vertical distance between the lowest and highest point of the groove in another direction; $\phi_{w}$ is regarded as the acute angle between texturedirection and feed direction, and is shown in Formula 8.

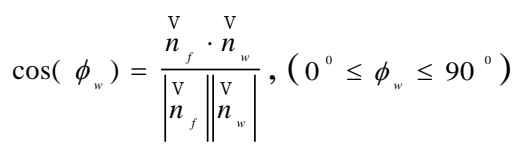

Where $\vec{n}_{f}$ is the unit vector in feed direction; $\vec{n}_{w}$ is the unit vector in texturedirection.

The nonuniformity of surface texture is expressed as the length difference of surface micro-units in the perpendicular direction of surfacetexture. The conditions of adjacent cutting teeth are different at a tool-rotation period when the errors of cutting amplitude and tool setting are less than the critical values. Different lengths of micro-units in feed direction will generate on the surface, leading to nonuniform surface texture in that direction.

Periodic variation of tool-setting error results in periodic variation of nonuniform surface texture in the cutting process. Cutting amplitude is affected by factors of work-pieces such as nonuniformhardness distribution. Thus there is no clear periodicity on thenonuniform surface texture caused by cutting vibration, and its texturevariation is periodic only in a certain range.

The direction of surface texture is the same with that of path interval direction if the maximumresidual height in feed direction is greater than the one of path interval direction, 
and the surface texture will be nonuniform. The uniformity of surface texture is the difference between the max-width and min-width of surface texture in the perpendicular direction of surface texture.

$$
\Delta l=l_{\max }-l_{\min }(9)
$$

Where $l_{\max }$ is the maximum length of micro-units in the perpendicular direction of surface texture; $l_{\min }$ is the minimum length of micro-units in the perpendicular direction of surface texture.

Constraint condition is that $\Delta l$ equals or less than $\Delta l_{\max }$, where $\Delta l_{\max }$ is the allowed maximum uniformity of surface texture.

\subsection{Prediction and Control ofMachined Surface Morphology:}

Mathematical model of surface morphology, in high-speed cutting of hardened steel, was the path model established through the processing path of any point on the cutter edge. The interaction of cutting edge and work-pieces formed many micro-units which were arranged to form the cutting surface structure and the machiningsurface morphology. Besides, the parameters description was realized for morphology characteristics such as micro-units and texture of surface morphology. The specific modeling procedure is shown in Figure 3.

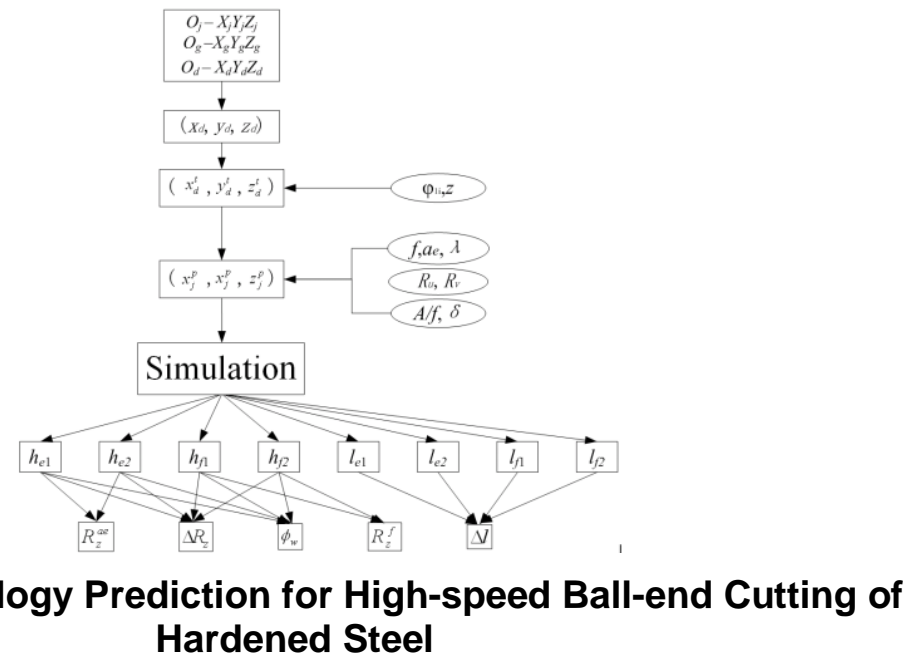

Figure 3 shows that $O_{j^{-}} X_{j} Y_{j} Z_{j}$ is the machine coordinate system; $O_{g^{-}} X_{g} Y_{g} Z_{g}$ is the work-pieces coordinate system; $O_{d}-X_{d} Y_{d} Z_{d}$ isthe tool coordinate system; $\left(x_{d}, y_{d}, z_{d}\right)$ is the coordinate of any curvilinear of cutting edge on the ball-head; $\left(x_{d}^{\prime}, y_{d}^{\prime}, z_{d}^{\prime}\right)$ is the coordinate of one point on cutting edge at the $j^{\text {th }}$ tooth when time ist $;\left(x_{j}^{p}, y_{j}^{p}, z_{j}^{p}\right)$ is the path of one point on cutting edge at feed of $i^{\text {th }}$; $\varphi$ liis the starting angle between $X_{d}$ axis and the feed direction of the first edge in $i^{\text {th }} ; n_{z}$ is the teeth number; $(A, f)$ is the cutting vibration amplitude and frequency; $\delta$ is the mount error of tools; $f_{z}$ is the feed of a tooth; $a_{e}$ is the path interval; $\lambda$ is the machining inclination angle; $R_{u}$ is thecurvature radius of work-pieces in the direction of $u ; R_{v}$ is the $v$ curvature radius of work-pieces in the direction of $v$.

\section{High-speed Cutting Experiment of Hardened Steel Dies}

Automobile skylight that is an outer panel is large in size and requires high machining precision and high-quality machined surface. And the processing of its die also needs high machining precision and high-quality of the surface is shown in Figure 4. 


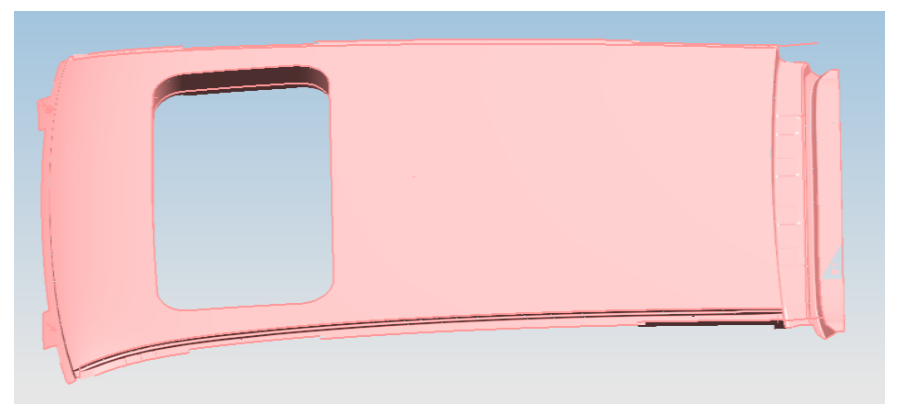

Figure 4. Car Sunroof

Table 4. Surface Features of Car Skylights

\begin{tabular}{c|c|c}
\hline $\begin{array}{c}\text { radius of curvature in main } \\
\text { profile }(\mathrm{mm})\end{array}$ & $\begin{array}{c}\text { height of side } \\
\text { elevation }(\mathrm{mm})\end{array}$ & cornerradius $(\mathrm{mm})$ \\
\hline 783 & $42-54$ & 50 \\
\hline
\end{tabular}

The surface features of the die of skylights are shown in table4. The optimization parametersof skylights, based on the die surface features, were obtained through the morphology control method of machined surface where spindle speed was 6000rp, feed rate was $4800 \mathrm{~mm} / \mathrm{min}$, path interval was $0.3 \mathrm{~mm}$, cutting depth was $0.2 \mathrm{~mm}$, tool was D30R15, and cutting style was that parallel processing was utilized on main die surface, equal-height processing on the sides and edges.

In this work, the quality of surface $(R a$ is $0.98 \mu \mathrm{m})$ and cutting area of unittime $\left(1200 \mathrm{~mm}^{2} / \mathrm{min}\right)$ were obtained. The surface roughness, compared with original process parameters, reduces to $30.6 \%$ of the original value, and processing efficiency increases by $33 \%$.

\section{Conclusion}

1.Based on interpretative structural modeling, we obtained a hierarchicalorder of evaluation indicators on high speed milling of hardened steel.

2. We established a model that predicted and controlled surface morphology in high-speed ball-end cutting of hardened steel.

3. By compared with the experimental results, the validity of predict and control method was verified.

\section{References}

[1] B. Yan, D. W. Zhang and An-ping Xu, "Journal of Computer Aided Design \& Computer Graphics", vol. 2, no. 13,(2001).

[2] J. M. Chen, "Simulation and Experimental Study on Surface Profiles Machined by High-speed Milling", Nanjing University of Aeronautics and Astronautics, Nanjing(2002).

[3] F. L.Feng, "The Nexus of Influence Element for Turn Cutting Machined Surface Roughness",Shanghai Jiao Tong University, Shanghai(2008).

[4] Tong Gao,"Numerical Simulation and Analysis for Machined Surface Morphology in Cutting Process and Form Error in Face Cutting Process", NorthwesternPolytechnical University, Xi'an(2004).

[5] X. S. Chen and C. R. Zhang,"Tool Engineering", vol.1, no. 41,(2007).

[6] X. F. Yu and J. W. Yu,"Journal of Hefei University of Technology(Natural Science)", vol. 1, no. 19, (1996).

[7] X. M. Li and N. Ding,“Chinese Journal of Mechanical Engineering”, vol.3, no. 43,(2007).

[8] S. R.Ge,Tribology, vol. 1, no. 17,(1997).

[9] C. G. Li and S. Dong, “Journal of Astronautic Metrology and Measurement”, vol. 4, no.18,(1998).

[10] B. L.He and E.Y. Shao,"Hot Working Technology", vol. 3,(1995).

[11] S. X. Zhu, "Study on Surface Texture Characterization and Friction Performance of Grinding. Nanchang University.Nanchang, (2007). 
International Journal of Control and Automation

Vol. 7, No. 11 (2014)

[12] Y. C. Tang and J. H. Fan, Systems Engineering”, Tsinghua University press, Beijing, (2010).

\section{Author}

Songtao Wang(1974-), Postdoctor,School of Mechanical and Electrical Engineering, Harbin Institute of Technology. hlgwst@sina.com 